\title{
THROMBOSIS *
}

\section{LUDWIG ASCHOFF}

FREIBURG, I. BR.

In the year 1911, at the meeting of the Deutsche Naturforscher und Aerzte, I submitted a review on the subject of Thrombosis. The question was brought up because the increasing number of operations in surgical and gynecological practice has made a fatal embolism of the lung, the sequel of a thrombosis, of more and more frequent occurrence.

The more we try to obviate this dangerous complication, the more urgent is our obligation to find out the cause of thrombosis. Now, there is no doubt at all, to use a mathematical figure of speech, that thrombosis is the function of a number of variables. There is not a single cause, but quite a number of different conditions which are closely related to the occurrence of thrombosis. Among these may be mentioned here, first, changes in the blood-plasma (diminished or increased coagulability); secondly, changes in the blood elements (increased or diminished powers of agglutination); thirdly, changes in the blood flow (slowing and formation of eddies), and lastly, changes in the vessel wall itself (endothelial damage). An inquiry into the mechanism of thrombosis shows that sometimes one factor, sometimes another, plays the principal rôle.

The view that increased coagulability of the blood is an essential point for the production of thrombosis, has been strongly upheld, especially by clinical observers. 'The existence of this increased coagulability, and the likelihood that it is a promoting factor, or, better, an accompanying phenomenon of thrombosis, cannot be denied. But all histological research since the early work of Zahn, Eberth and Schimmelbusch, Welch and other's, speaks for the view that in human beings the occurrence of fibrin coagulation is not the first stage of thrombosis, but that important changes in the morphological blood constituents precede it. 'These latter changes must be explained before the mechanism of thrombosis can be understood.

It is concerning the morphological structure of a thrombus, in continuation and amplification of my review mentioned abore, that I wish to speak.

\section{MORPHOLOGICAL STRUCTLRE OF THROMBI}

Since we have known from the work of Zahn, on the one hand, and of Eberth and Schimmelbusch on the other, that the blood-platelets and leukocytes really have to do with the building of a thrombus, the idea

* The Cartwright Lectures for the year 1913, of the Association of the Alumni of the College of Physicians and Surgeons, Columbia University. 
readily suggested itself that the whiter color of one part of a thrombus was to be referred to an accumulation of these elements, which would thus appear to constitute the first material laid down in the process. We have become accustomed to speak of white, mixed and red thrombi, but the relationship of these different appearances to one another, in spite of the early work of Zahn and others, has not been wholly and correctly appreciated in the literature of the subject. We must remember with Welch - to whom we must give thanks, as well as to the previously mentioned authors, for the most exhaustive experimental work on thrombosis - that in a completely finished thrombus, for example in the femoral vein, the variations in the colors are perfectly regular. The first part (Kopfteil) of the thrombus is chiefly of a white color, and represents the so-called white thrombus, and on this a middle part (Halsteil), mixed in color, and a deep red, distal and final portion (Schwanzteil) are subsequently laid down. The first part (Kopfteil) may be of the smallest possible size and extent; whereas the final red part (Schwanzteil) often forms the bulk of the thrombus, and may measure many centimeters. Thrombi in other parts of the venous system are built up on exactly the same principle.

It is easy to understand that red thrombi occur only where a white thrombus has more or less completely obstructed a ressel, and that they cannot occur where the white thrombus only partially obstructs the lumen.

Very long white thrombi can arise only when the blood-stream continues to pass through a vessel. From all these points a rule can be formulated which holds for the majority of all autochthonous thrombi in human beings; namely, that the white thrombus is the determining and peculiar factor in the whole process, and that red thrombi are only, so to say, accidental-they may occur but not necessarily. An exception would of course be the case of a pure red thrombus arising by autochthonous thrombosis, and not consisting of a piece of an ordinary thrombus which had been broken off and carried away. Such an event, if it occurs at all, is certainly very rare.

Our interest, then, centers on the question of what this Kopfteil, or first part of a thrombus, looks like under the microscope. The structure of it will perhaps give us an understanding of its mode of origin.

First of all, we must remember that the much quoted Zahn's markings on the outer surface of thrombi-the small ripple-like, net-like and linear markings - are only to be seen on the Kopf and Halsteil of thrombi. whereas in the red part of the thrombus they fade and soon totally disappear. These markings are merely fine, white elevated lines or ridges which are specially well brought out when the furrows between are reddish in color, or where a mixed red and white thrombus, as in the Halsteil, is present. Along with the explanation of this marking stands or falls the 
whole problem of thrombus formation, so far as consideration of the majority of cases of autochthonous thrombosis goes. This decision appears at first sight a bold one, but will readily be understood when the microscopic structure has been taken into account. A longitudinal section through the Kopf and Halsteil of a thrombus shows that the very delicate surface elevation is only the summit of a framework of beams, which in delicate rings like a mass of coral, forms the skeleton of the whole thrombus. When a thrombus at quite an early stage in its development is examined, this framework is seen to be a finely granular mass which consists simply of an accumulation of blood-platelets. All the beams of this framework are surrounded by a border of polymorphonuclear leukocytes, by means of which they are differentiated even more sharply from the red blood-mass which fills up the numerous spaces between.

But it is not only the regular and definite separation of the different blood-elements which is surprising, but the further fact that all the beams of the framework stand in a very special relationship to one another. The beams follow one another at fairly regular intervals, and build up group-like systems, inside of which the direction of the beams is much the same. In addition, however, secondary beams are seen in these groups extending either upwards or downwards from the primary beam of the framework. It must be admitted that dissimilarities also occur here. The more we approach the pointed outer extremity of the Kopfteil the broader become the beams of the framework, the furrows between disappear, until finally the beams unite into a single mass, to form the pure white outer surface of the Kopfteil.

The most important point, however, in the structure of this whole system is that fibrin practically does not appear. When it is found, however, the strictest regularity governs its situation. The threads of fibrin shoot out first of all along the borders of the framework of bloodplatelets just where the red blood touches the framework. Subsequently it penetrates more and more into the blood itself. A structure such as has just been described is the only sure token by which we can recognize microscopically the intravitam origin of a thrombus.

With this explanation of this structure, we are enabled at the same time to understand the way in which a thrombus is built up. The explanation involves the solution of a complicated physical problem, which I can present here only in a general way, since, in spite of many discussions with my colleagues in the department of physics, I have been unable to get to the bottom of it. What the vital question is, we can state nowadays with certainty. This question is: Does the thrombus arise in the flowing or in the stationary blood-stream? So long as the view was held that white thrombi were built up from leukocytes, as Zaho pointed out, it had to be assumed that it was only in the flowing blood 
that these thrombotic masses could be laid down. Zahn himself Iaid great stress on this point. But when it became practically certain that a thrombus takes origin exclusively from blood-platelets, uncertainty again arose, since the origin and meaning of these platelets was veiled in obscurity. While some observers affirmed the independence of these structures, many more considered them to be merely distintegration products of white and especially of red corpuscles. If the latter theory were true, a thrombus could quite well be considered to be formed from a small mass of dead and disintegrated red blood-cells, which had died because of the stopping of the blood-stream. But it could not explain why the heap of particles at once proceed to build themselves up into such a beautiful framework and divide themselves off so sharply from the red corpuscles.

Taking it for granted that the origin of blood-platelets is not from leukocytes or erythrocytes, but that they are independent elements existing by themselves in the blood-streams (as indeed the excellent researches of Deetjen have already suggested), it becomes quite clear to us that such accumulations of blood-platelets as occur in a thrombus can be deposited only when the blood is circulating. The important question of the origin of the blood-platelets can, in my opinion, now be regarded as settled. They take origin neither from white nor red corpuscles, but are, for so long as they circulate in the blood, independent structures. J. H. Wright of Boston was the first to point out their origin from the giant cells of the bone-marrow and spleen. In his important researches he was able completely to confirm the statement of Schridde that the megakaryocytes have a finely granular protoplasm except for a zone at the margin, which remains clear. He then showed that from the giant cells small portions are nipped or budded off in the form of small platelets, each of which consists of a finely granular central portion surrounded by a clear marginal zone. These are in fact the blood-platelets, and the granular center, the origin of which is thus readily understood, is identical with the structure often described, especially by Deetjen, as a nuclear structure in the platelet. That independent movement is possessed by these structures had already been shown by Deetjen. Wright's findings, doubted on many sides, were reinvestigated by Ogata in the Pathological Institute in Freiburg, and were all completely confirmed. In fact in fresh, warm material taken from different animals for investigation the budding off of platelets from the megakaryocytes can be plainly followed. At the time of the budding-off process the protoplasm of the giant cell is seen to have invaginated itself into a capillary in the form of a pseudopod; and thus the occurrence of the platelets in the blood-stream comes about. 
The independence of the blood-platelets in the circulation can be proved in another and very positive way. If they arose simply by the disintegration of red corpuscles, then the destruction of a stationary column of blood, as can readily be produced in a doubly ligated vessel by the corrosive action of a small piece of silver nitrate, would be followed by a blood-platelet thrombus-building, just as occurs in the flowing blood-stream where a typical thrombus forms on a damaged piece of vessel wall. This question was investigated by Derewenko ${ }^{1}$ in the Pathological Institute in Freiburg with striking results. He found not a trace of heaping-up of platelets in the doubly ligated vessel in spite of all the damage, whereas in a vessel through which the blood still flowed a typical thrombus had been built up from blood-platelets, showing the characteristic framework we have learned to recognize in the human subject.

We have concluded then, that the thrombus arising from the bloodplatelets has its origin in the circulating blood-stream, and the question yet to be answered is: Why do the platelets build themselves into this peculiar framework? To this important question I can give no final answer. A few interesting points only may be raised. By the making of serial sections of human thrombi, and subsequently building up models, Ferge, ${ }^{2}$ working in the Pathological Institute in Freiburg, was able to determine with certainty that the platelets do not form beams running in circles but do form a system of lamellae, more or less parallel and arranged on the vessel wall one behind another. Most of them rur obliquely or transversely to the long axis of the vessel. These lamellae grow outward from the vessel wall, and from the flow of the blood through a part of them, they become bent in the direction the blood is flowing. The appearance of this system is strikingly similar to the beautiful figures made on the fine sand of the seashore by the ebbing tide. The water pushes up the fine sand into ridges, until the strength of the flow overcomes the small obstacle, and the water travels on to build new ones further on. In this way then the beautiful series of ripple lines are drawn on the shore. The blood-stream builds up its lamellar system in an exactly similar way from the sand-like multitudes of blood-platelets. Zahn had previously taken notice of the work of de Candolle on the curious sand formations produced by moving water, and had put forward the suggestion that the lines visible on the surface of the thrombus are simply the effect of to and fro movements of waves of blood. But the essence of the matter is not that already existing sandy masses are shaped into systems by the movements of the blood, but that solid elements are taken from the flowing blood-stream and laid

I. Derewenko: Zieglers Beitr., 1910, xlviii, 123.

2. Ferge: Med.-naturw. Areh., 1909, ii, 351. 
down in the form of specially situated lamellae. I can only mention here in passing that by experiments which I carried out in the laboratory of Professor Rehbock I was able to find exactly similar deposits formed in running water from a given solid material, when the velocity of the flow was reduced sufficiently by the introduction of a dam or weir. No transformation of already laid down masses of particles was necessary to make the ridges, but the particles were laid down in such a way from the first. The question, of course, arises from these experiments as to why a deposit of platelets occurs at all, and this will be discussed later on. So long as the blood flows through this framework just as sea water flows beneath branches of a tree of coral, new masses of blood-platelets are laid down on the system already formed, and so new systems are always being built up behind the first one. The first formed system naturally grew faster, and finally there comes a time when the openings in the lamellae of the old part of the thrombus (this is indeed the Kopfteit which has already frequently been referred to) are so narrowed that the blood-stream becomes slower and slower, and finally stops altogether. With this event the building of a primary thrombus is finished, since once the blood-stream is at a standstill no new blood-platelets can be carried past, and hence further growth is impossible.

But before the complete cessation of the blood-stream occurs, a certain peculiar separation of the red and white corpuscles comes to pass, resulting in the whole system of platelets becoming covered with a layer of leukocytes. The facts of this peculiar separation are best explained by the well-known researches of Eberth and Schimmelbusch, who found that when the blood-stream was slowed the white corpuscles being of lighter specific gravity than the others, tended to travel at the margin of the blood-stream, and so are found closely in contact with the vessel wall. By the growing system of platelets which occurs in the formation of a thrombus the main blood-stream is divided up into a series of small streamlets. Each small streamlet is, however, subject to the same laws as a larger vessel, and when the slowing of the blood-stream occurs, the leukocytes are found close to the sides of each small streamlet, whose wall is in this case composed of the framework of blood-platelets.

Before I can turn to the question of why the blood platelets are deposited from the circulating blood, I must briefly describe the results which follow in the peripheral part of the vessel, when the stream is completely interrupted through the gradually increasing obstruction of a system of platelets. As soon as the lumen is closed in the region of the white, that is the primary thrombus, the whole blood-column becomes stationary right up to the place where the next anastomosing vessels enter, and undergoes very rapidly, as experiment shows, a complete coagulation. In this Schwanzteil of a thrombus, which is usually of soms 
length and like the rest in color, we can expect, it goes without saying, no definite framework of platelets, and in fact it is either completely absent or occurs only partially. This red thrombus resembles in its microscopic structure essentially a post-mortem clot, and consists of an irregularly arranged mass of red and white corpuscles, blood-platelets and fibrin. It must be admitted that here the leukocytes and bloodplatelets have a tendency to form themselves into masses, but there is no attempt at the formation of lamellae. The fibrin frequently shows striped thickenings running in definite directions. These may be the results of currents in the plasma, for although the column of blood has been checked it is still connected in manometer-like fashion with the rest of the vascular system. They may also merely be the expression of the movements of the fluids induced by the coagulation.

Thus an essential difference exists between the Kopfteil and Schwanzteil of the thrombus, which it must be said are connected together by a more or less transitional zone. This difference we know must depend on the fact that the Kopfteil is formed in flowing blood, the Schwanzteil in blood at a standstill. This brings up the question then of why a white thrombus is built up from platelets in the flowing stream and a red thrombus when the blood-flow has ceased.

\section{MECHANICS OF THROMBUS FORMATION}

After the corpuscular nature of white thrombi was recognized by Zahn, von Recklinghausen directed attention to the important bearing the slowing of, or rather the formation of eddies in the blood-stream, had on this question. His work has given considerable support to the wellknown teaching of Virchow regarding the mechanical genesis of thrombi. When we compare the blood-stream with a river which has countless small particles of different specific gravity swimming about in its waters, we are driven to the conclusion that there must he some optimum velocity which will bring about a local aggregation of particles, such as we have seen by the microscope to occur in the building of thrombi. Neither by a rapidly flowing stream nor by complete stoppage of the current can such a heaping up be brought about. Thus the blood must flow slowly, or, to put it in a better way, must flow differently from the way it did before. It is noteworthy that deposits of sand in a river occur by preference where a widening or a deepening of the river bed exists. It is not a uniform slowing, but the inequality of the local conditions which predisposes to this extraordinary deposit. The insertion of a dam or barrier, by introducing counter currents and eddies, is also an important predisposing cause. I have made an effort to explain this building of sand banks on purely physical lines, and in this I have had the assistance of Prof. Dr. Rehbock in whose laboratory the experiments were 
carried out. If a weir is introduced in a stream of fluid, then by the interference with the current so induced an unusually long "Walze" (or helix), as it is called technically, is produced in which a portion of the fluid flows backward. And not only that, but fine particles suspended in the water (we used sawdust for the purpose) all deposited in ripple

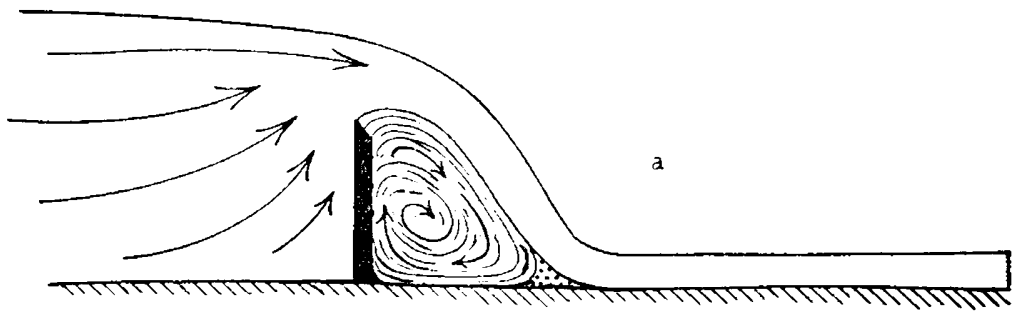

b
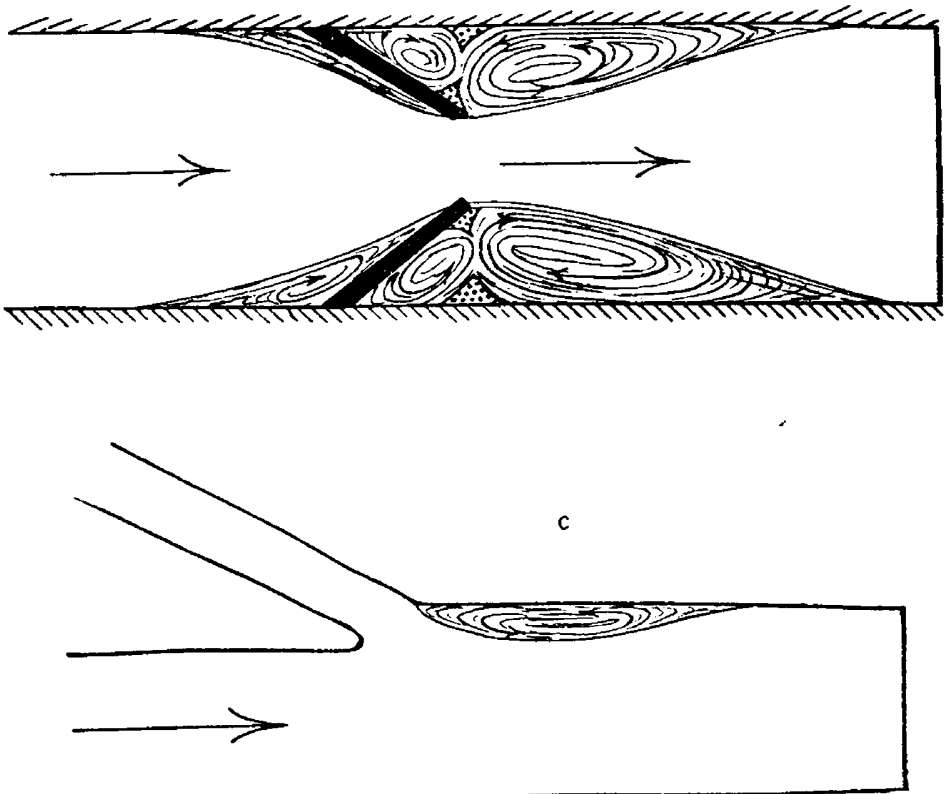

Diagrams showing (a) eddy formation behind a weir; (b) eddy formation in front of, behind and beneath obliquely-placed weirs; (c) eddy formed at the point of junction of two streams of unequal size. (From Aschoff: Thrombose und Sandbankbildung., Ziegler's Beitr., 1912, lii, 209.)

and net-like elevations in the regions of this "Walze." By means of obliquely placed weirs two "Walzen" can be produced, the one proximal, the other distal to the obstruction. It is at the point of contact of the two that thrombi arise, and, as Ferge also found, the thrombus is not built close under the valves of the veins. (See Fig. 1.) 
Finally the question of sand-bank building by the flowing together of two unequal streams must be considered from the physical standpoint. When we study what occurs in a pipe when this state of affairs is brought about, it will be found that a slowing of the stream in the so-called zone of transition occurs. If the stream from the smaller vessel flows very slowly a sand-bank is formed at its mouth. This agrees very well with what is found in human pathology.

All these experiments are, on account of the number of factors outside our control, merely suggestive. We can take no account of the part played by the living blood and the living vessel wall. We can only prove the gross results and ask what alterations of the blood-stream, in the form of sudden slowing, backward flowing and eddy making, are found in the places where in the human subject autochthonous thrombi have a tendency to grow.

\section{LOCAL CONDITIONS FAVORING THROMBOSIS}

The special tendency to slowing of the blood-stream is quite properly insisted on by every one who has written on the subject. It is quite clear that some very special and unusual condition must be present in the arterial system to slow the blood long enough to allow a deposit of blood-platelets to occur. As Virchow pointed out long ago, and as is now generally recognized, there are certain situations in the venous systems which are especially predisposed to thrombus formation. Among these the veins of the leg, the proximal part of the femoral vein where the large valves are present, the pelvic plexus, the venous network of the dura mater and the auricles may be taken as examples. There are besides four conditions each of which, alone or in combination, has to do with the localization of thrombosis. First, there is continued overpressure on the wall of a vein, such as occurs in the veins of the leg from the pressure of the column of blood when the body is upright, or such as is brought about in the renous plexus of the pelvis by downward pressure of the intestines. This overpressure tends to physiological widening, and, finally, may terminate in a physiological thrombosis. I refer to thrombus formation in varicose veins in the lower limbs and to the thrombosis in the prostatic plexus in old age which is so usual as to be practically physiological. 'Thrombus building in the vaginal plexus, which may be the origin of a spreading thrombosis or be a nidus of secondary infection, has not had sufficient attention paid to it.

A second condition is the widening which obtains in the auricles, and also occurs in the veins at each valvular sinus. Ferge's recent experiments have confirmed the work of Kölliker and Epstein, who discovered that the musculature of the vein wall can be practically absent in the region of the valvular sinuses, so that backward pressure must 
be followed by an ampulla-like widening above the valve. Further, the possibility of a backward pulsation in the veins, the so-called venous pulse, which often is most marked at the proximal end of the femoral vein, must not be lost sight of. Lastly, when the body is lying prone, certain local conditions affect directly certain veins with a known tendency to thrombosis. For example, the femoral vein just where the large valves are present, lies close under Poupart's ligament, and the importance of the bend so brought about must not be underestimater. The iliac vein shortly after the junction of the hypogastric vein, also comes into the same category, and its tendency to thrombosis is also to be referred to the bend in the path in which the blood flows.

Naturally, these local factors play an even greater part when any defect in the heart's action leads to a general slowing in the venous system. In such a case the posture of the patient exerts a further and additional influence. In certain conditions the posture has directly to do with the left or right sided position of the thrombus, the limb which lies lower having a greater tendency to become thrombosed. When lying on the back the increased compression of the left iliac vein by the arterial trunks (right iliac, middle sacral and left hypogastric arteries) has a direct influence in slowing the stream, and explains the well-known frequency of thrombosis in the left lower limb. Another point, too, is of interest. When a double sided thrombosis of the veins of the lower limbs occurs, the following characteristic conditions can often be found. The thrombosis on the right side extends up to Poupart's ligament, whereas on the left side it extends up to the point of compression of the left iliac vein by the right iliac artery.

The deposition of blood-platelets in all the above mentioned examples of thrombosis is easily understood. It is not the stagnation but the retardation which brings about the thrombosis, and must be reckoned as the direct cause of the deposition. Eberth and Schimmelbusch have given to this type the name of conglutination thrombosis. They took this descriptive name from a phenomenon which occurs in the heaped-up mass of platelets, namely, the cementing of the platelets together. This phenomenon can also quite truly be called agglutination, so the term agglutination thrombosis is also a suitable one.

Between accumulation of platelets on the one hand and conglutination or agglutination on the other there exist very special relationships.

We do not know as yet anything of the phenomena which precede agglutination and which are the very earliest factors in thrombosis. We cannot say whether they are of a type long known to workers in bacteriology or serology, or whether they are chemicophysical. We suppose at least that a special degree of viscosity must be present to allow the masses of platelets to form so easily, whereas precipitation phenomena 
in the sense of agglutination can also play a rôle. It is also quite conceivable that a mass of platelets, once it is formed, can exert an influence on other platelets flowing past it. The agglutinability of the platelets depends, of course, on the chemicophysical characters of the fluid in which they are, in this case, the blood-plasma.

Thus we see that alteration in the blood-stream is only one condition in the making of a thrombosis, the second condition being dependent on the agglutinability of the platelets themselves. Unfortunately, we have no knowledge of spontaneous changes occurring in this property of agglutinability. This question, chiefly from the experimental side, is considered by Achard and Aynaud.

It is evident that the number of the platelets is an important matter to be considered in relation to thrombosis. Judging from the literature it seems clfar that the number of platelets-their exact enumeration is no light matter - undergoes great variations in cachectic states. We know too, that after experimental anemia the number of blood-platelets increases rapidly, coincident, as has been shown by Ogata, with increase in the number of giant cells in the marrow. It is, therefore, quite feasible to suppose that subsequent to loss of blood at operations or during parturition, an increased tendency to thrombosis can be brought about, although it cannot at all be said that an increase in the number of platelets can by itself alone bring on thrombosis.

\section{VASCULAR CHANGES AS INFLUENCING THROMBOSIS}

We have up to this point considered two of the conditions which have to do with the building of thrombi, namely, changes in the blood-stream, and qualitative and quantitative alterations in the platelets. We will now take up a third condition, which used to play the chief rôle in the teaching about thrombosis, but whose signification was soon greatly limited by Virchow. I refer to alteration of the vessel wall itself. That this is not the decisive, not to say the only cause of thrombosis of the ordinary type, is made very clear when we can think of an atherosclerotic aorta, which in spite of the most marked changes can remain quite free from thrombi. Here, of course, there is no slowing of the blood-stream. On the other hand, the question arises whether the slowing of the stream can alone, without any accompanying change in the vessel wall, give rise to a thrombosis by deposition. Do the deposited platelets remain bound together only when the endothelium is damaged? Is this damage of the endothelium a third condition which must be fulfilled before thrombosis by deposition can arise, and how must the endothelium be damaged? Covering this point of endothelial damage, the importance of which is always accepted without question, and which is always given great prominence in the literature, we know in reality practically nothing. We 
can affirm only one thing, namely, that chronic changes of slight degree in the intimal lining of a vein, as for example fatty changes of the endothelium, can by themselves play no important rôle, since one must so often assume this change to be present without thrombosis resulting. Besides, it is very difficult to understand how a fatty change of the endothelium merely can lead to a deposition of blood-platelets. I can find in the literature no well authenticated cases, in which thrombosis has really been brought about by changes in the endothelium, a factor which is regarded by many authors as of special importance. It is naturally quite another story when rigidity of the wall and valves of the vein, brought about by severe and progressive phlebosclerosis, has interfered with the blood-flow. Changes in the wall of the vein are then not the direct, but only an indirect source of thrombus formation. An analogous state is brought about in the numerous experiments carried out to induce thrombosis by means of corrosives, cutting and the introduction of threads or other foreign elements. These experiments were carried out at an early date by Zahn, Eberth and Schimmelbusch, Welch, and more recently especially by Zurhellen in the institute in Freiburg. Here the rough and mechanical disturbances of the blood-stream resulting from the laceration of the vessel wall, do not play the entire rôle, but the plasmatic exchanges between the fluids of the blood and the lining of the vessel must also be important. When instead of slow retrogressive changes, the intima is suddenly stripped of its endothelial lining, then a reaction inevitably ensues, as occurs in all living tissues, accompanied by a pathological flow of lymph. Then it is, indeed, the alterations in the blood-stream in the region of the injured area which bring about a thrombosis by deposition. It is not made clear by the previous experiments how and whether a direct influence on the bloodplatelets as they flow by can possibly be exerted by agencies of an agglutinative or coagulative nature produced by the tissues ( L. Loeb, Achard, Aynaud). Nor is it explained whether the connective tissues or the muscle laid bare by the damage at a surface capable of absorption (Morawitz) can bring about the adhesion of the platelets. At any rate, all these artificially produced thrombi present the characteristic structure of thrombi by deposition of the blood-platelets. We must allow that up to the present we have not been well enough informed concerning the first and absolute beginnings of thrombus formation in the case of autochthonous thrombi in the human subject. Unfortunately in this connection we can hardly ever obtain fresh enough material, since, of course, we must eliminate all post-mortem coagulation, which naturally can occur in the thrombus itself.

We must endeavor to get a clear idea of why the adhesion of the platelets to the vessel wall occurs in autochthonous thrombosis, since we 
assume that this deposition of platelets is the first procedure. It may be quite possible that the endothelium dies as a consequence of being covered over by the platelets. Fibrin is in this way set free and cements the masses of platelets to the wall. Or the platelets after lying on the wall for some time may themselves die, and then coagulation on and between the endothelial cells can ensue. At any rate, it is necessary that an interaction of a certain duration occur between the platelets and the endothelium in order that the material may remain stationary and, so to say, be fixed there. If slowing of the blood-stream and alteration in the conditions of the platelets are to figure as the direct facts in thrombus formation, then we must consider as indirect factors changes in the wall, alterations in the cardiac action and loss of blood during operations.

In considering the vital question, as to why a white thrombus occurs in the circulating blood, and a red thrombus only when the stream is stationary, I have already attempted to answer the first part and shall now proceed to the second. I have already mentioned that a red thrombus arises only when the lumen of the ressel is sufficiently closed by a white thrombus to bring the blood-stream to a standstill in the peripheral portion of the vein. This stationary column of blood coagulates. But under the microscope this red thrombus, dense in the neighborhood of the Kopfteil, while peripherally becoming more and more spongy, and, finally, almost fluid in consistence, can scarcely be differentiated from postmortem clotting. The microscope shows, however, that the denser portion is richer in fibrin and leukocytes, and suggestions of lamellae can here and there be recognized. As we travel more to the periphery the structure of the thrombotic mass more and more approaches that of the normal blood. Microscopically, it is also true that the boundary between the white and the red portions is as hard to recognize as that between the slowing and final complete stoppage of the blood-stream. From these points we can also explain why this column of stagnating blood does go on to coagulation, in contradiction to the well-known findings of Baumgarten, who observed that the column of blood contained in a doubly ligated portion of a vessel did not coagulate. We must take into consideration that, as has been already mentioned, coagulation phenomena can also be observed to have taken place in the blood found occupying the gaps in the white portion of a thrombus. These phenomena very probably must be referred back, judging from the characteristic situation of the fibrin network, to an interaction between the fibrin ferment set free by the death of the platelets and the stationary blood-plasma round about them.

Of course, the amount of fibrin ferment set free in the white Kopfteil will be very considerable. It can advance by diffusion into the caudal red portion of the stagnating blood-column, which is richer in leukocytes 
and platelets and develop its action. We know, as Baumgarten has stated, that the cause of the blood remaining fluid in a doubly ligated segment is that the blood dies relatively slowly. Conversely, we can presuppose a very rapid death to occur in a structure rich in ferment, as the blood-platelets are, and so the clotting of a column of blood in stagnation becomes intelligible. I will not consider here the processes which precede clotting, and above all I cannot discuss the question of where the forces which bring about clotting have their origin. Nor can I stop to inquire whether the source of thrombokinase is to be sought for only in the thrombocytes and leukocytes, or in the erythrocytes as well. I would prefer to refer you to the well known and very clearly expressed papers of Morawitz. ${ }^{3}$

I pass over here the finer morphology of the processes preceding clotting, and would only mention that the "centers of coagulation," so-called, are to be regarded in my opinion as acting both chemically and physically. In the origin of the red thrombus we are also dealing not with the separation of the formed elements of the blood, which practically does not occur, but more especially with crystallization of fibrin as in ordinary clotting. Thus we have a coagulation of the blood as a whole, and not merely the deposition of some parts of it. This view has already been suggested by previous authors, who have put forward the name coagulation thrombosis, in contra-distinction to conglutination and agglutination thrombosis.

Just as in deposition thrombosis the slowing of the blood-stream and the relations of the platelets as to number and viscosity are the essentials, so in clotting "en masse" the stoppage of the stream and the increase of fibrin ferment are the important factors. It cannot be denied that an intimate relationship exists between the heaping up of platelets, the agglutination and the clotting, i. e., the coagulation. He must conclude that agglutination is, so to speak, only a means of creating in the circulating blood conditions which are necessary to allow of the crystallizing out of fibrin, a process which cannot occur in the circulating blood under ordinary conditions.

But it is quite evident that these two phenomena (agglutination and crystallizing out of fibrin) do not mean the same thing and must be kept genetically apart, since each can occur without the other. In the case of ordinary autochthonous thrombosis, with which we have been dealing up till now, the deposition and coagulation processes are closely related. The process of deposition brings about the coagulation, and is, so to speak, the indirect source of it. Then the further question arises as to whether coagulation thrombosis can occur apart from the process of deposition, and what may be the indirect causes influencing them both.

3. Morawitz: Blutgerinnung, Handb. d. Biochemie d. Menschen u. d. Tiere, 1908. 
It is important in this connection to remember that while thrombosis by deposition can be brought about readily at any time, a primary coagulation thrombosis is very difficult to induce. The stoppage of the blood stream alone does not suffice, as Baumgarten's experiments have shown, for the ferment does not develop quickly enough. Also optimal relations must be established between the rate of stream and amount of ferment present, to initiate the process of clotting. Even the flowing blood, however, can be made to clot, if a large quantity of ferment is introduced all at once, either by the injection of pure fibrin ferment or by artificial transfusion. Of course, control by the microscope is essential in such experiments to ascertain whether the solidification of the blood depends really on clotting of fibrin, and is not merely a precipitation, such as occurs when a substance which coagulates albumin is introduced.

To the question of these precipitation thromboses, and other related processes, I will return later.

Two other processes must be mentioned here which undoubtedly have relationships with coagulation thrombosis. I refer to ligature and infection. Since, as we shall see, both of these can also induce thrombosis by deposition, we can now inquire how ligature and infection, respectively, act, and what is their significance from the point of view of thrombosis.

The facts in the case of ligature are comparatively simple. If done carefully so that considerable damage to the intima is avoided, and the portion included by the ligature is so small as not to interfere with the movement of the blood, no clotting need necessarily occur, a fact which. we have noticed elsewhere in connection with transplantation of bloodvessels. At other times we find thrombus formation, often quite scantily, however, in the region of the intimal damage, where the fibrin ferment arising from the injured wall can act on the stationary blood in its neighborhood. Or at the margin of the ligatured part of the vessel a true deposition thrombosis may arise as a result of local eddy formation. In this way the area may finally be separated off completely from the blood-stream, and the column of blood thus shut off passes into a condition of coagulation thrombosis, like the Schwanzteil of an autochthonous thrombus.

\section{RELATION OF INFECTION TO THROMBOSIS}

Much more difficult to explain is the question of infection to thrombosis, nowadays considered so important. It is especially from the findings of clinicians that the idea has been put forward that not only most, but as some will have it, al! forms of thrombus formation are to be referred back to infection. It is unfortunate for the advancement of pathological anatomy that when as a sequel of thrombosis cases come to autopsy, an infection often does not exist or at any rate can no longer be 
recognized. Concerning infective thrombosis, I would say only that from my own researches I am of opinion that what occurs generally is a secondary infection of an already existing simple thrombus; for example, in the site of an operation or in the area of uterine wall laid bare by the removal of the placenta. Of course, it is not impossible that a primary inflammatory process in the surrounding tissues can implicate and extend through the wall of a vein and a thrombosis occur from the changes in the bloed-stream arising in that way. Of more importance, however, than these locally infective thrombi are those of septic character which arise at a distance from the site of an operation; for example, septic thrombosis of the femoral vein following operative interference with the pelvic connective tissue. Here our observations have shown that a typical thrombosis by deposition always occurs, the organisms themselves being found enclosed here and there in the framework of the thrombus. One must necessarily conclude from this histological picturs that the organisms circulating in the blood are only, so to speak, secondarily enclosed in the thrombus while it is being built. The thrombus is otherwise identical in structure with a simple one. We must, therefore, assume that here the thrombosis has a similar origin as in the noninfective type, namely, a slowing of the blood-stream, and that the organisms themselves are not the causative agents. The latter are merely accidentally enclosed, but are dangerous, since by their multiplieation they convert a simple thrombus into a septic one, and may bring on all the consequences of septic emboli. The following abridged account may be given of the complicated picture which is produced by the infection occurring in the thrombus in a vein.

1. A local infective thrombosis in the region of an operation or in the neighborhood of the placenta can arise: (a) Through infection of thrombi already present, to which an inflammation of the wall has arisen secondarily (thrombophlebitis). (b) By an infection progressing along the vessel wall, which induces first of all a primary phlebitis and is followed by a secondary thrombosis by deposition (phlebitic thrombosis). (c) By means of slowing of the blood-stream and thrombus formation in the commencements of the veins followed by thrombosis by deposition in the larger veins, induced by toxic or inflammatory causes, which subsequently become infected.

2. An infective embolic thrombosis arising at a distance is in most cases the sequel of an ordinary primary thrombosis, which acquires its infective eharacter secondarily by multiplication of micro-organisms in the blood shut in the interior of the thrombus. Experimentally it would appear probable that only exceptionally the organisms separate themselves from the blood, directly infect the wall and so bring about a secondary thrombosis.

3. All septic thrombi can extend on account of the inflammatory changes in the wail. These are not confined to the area in contact with the clot, owing to the toxins being diffusible.

Since in the case of distant thrombosis the infection usually arises in already existing thrombi, and cannot be regarded as the source of the thrombosis, but rather as an accompanying or induced phenomenon, we 
must inquire why, especially after operative procedures or in already established infection, thrombosis at a distance is of frequent occurrence. It is well known that in acutely progressing septic cases thrombosis at a distance is much rarer than in subacute or chronic cases. That in itself speaks against a direct action of infection.

Thrombosis of the femoral vein, among the thromboses at a distance, interests us most, since it is the special origin of fatal lung emboli. Here extremely varying factors work together - loss of blood at operation, weakening of the heart, general prostration, confinement to bed, the influencing which every pelvic operation has on the return of the venous blood from the lower limbs to the heart, because the venous plexus of the pelvis acts as a manometer to this great area of blood.

Finally, the possible changes in blood platelets themselves induced by the infection must also be considered. In reality we have no positive knowledge on the question whether the bacterial toxins can increase the viscosity of the blood-platelets.

I have taken up so much time with the static or autochthonous thrombosis, because it seems to be the most important variety, and is, as a rule, easily recognized both by the clinician and the pathologist. With its description, however, the question of thrombosis is in no way exhausted. I have already made special mention of the work of my American colleagues, Flexner and Loeb, who, by using toxins, studied the processes in the living body which accompany the toxic thrombosis described previously by Naunyn, Landois, Ponfick, Silbermann, Kaufmann, Falkenberg and others, as resulting from blood transfusion and specific blood-poisons, and by 'Takowski, Lubarsch, Bardeleben, etc., as a consequence of injection of bacteria and bacterial toxins. Recently in Germany, Dietrich and my own pupil, Kusama, have carried out such experiments, and I would like to make a few remarks about Kusama's work which has only lately been completed.

\section{CAPILLARY THROMBOSIS}

Since the independent elements of the blood are influenced in the highest degree in all these thromboses of toxic or infective origin, while the conditions in the blood-stream itself remain essentially the same, I have called this form of thrombosis "thrombosis by alteration of the blood," in contradistinction to "static thrombosis." Further, as you will hear immediately, the initial thrombus building occurs in the capillary system, and so one can also call this form capillary thrombosis, in contradistinction to that occurring in the larger vessels.

In continuation of the work of Flexner and Loeb, who, by injecting heterologous serums, have explained the significance of the agglutination of erythrocytes, the deposition of platelets and the formation of fibrin 
in thrombosis, and in connection also with the work of Dietrich, who, above all, has tried to elucidate the mode of action of the end-products of hemolysis, namely, the blood-cells on one side, and the agglutinins and precipitins on the other, my pupils and I have tried to recognize the factors which are essential for the production of thrombi in the circulating blood.

So far, our experiments agree more with those of Flexner and Loeb than with those of Dietrich, who studied chiefly the effects on the stationary blood-column. We began by investigating the effects of homologous serums on rabbits. I shall take no notice here of the clinica! symptoms induced nor of the altered coagulability of the blood, but would chiefly confine myself to the morphological findings. Rabbits showing no signs of illness, were killed twenty minutes after the injection of a homologous serum. Examinations of the organs just after death showed in the places predisposed to capillary thrombosis - for example, the capillaries and post-capillary venules of the lungs - very abundant thrombus formation. The thrombi consisted in parts exclusively of blood-platelets, packed together but well preserved, in other parts of a mixture of platelets and red-cell stromata. When the fixation was good, fibrin was absolutely absent or only to be found in traces inside the thrombi. On what, then, do these thrombi, formed from platelets and red-cell stromata, depend? They might be a direct effect of the serum injected, which both agglutinated the platelets, and after hemolyzing the erythrocytes, agglutinated their stromata. To answer the question, Kusama hemolyzed red corpuscles by washing them thoroughly with distilled water and then injected the solution of hemoglobin. When the animals were killed, ten to twenty minutes after such an injection, thrombi composed of blood-platelets could be found in the vessels of the lungs. On the other hand no thrombi composed of stromata were met with. A strongly agglutinative effect on the blood-platelets is thus exercised by hemoglobin circulating free in the plasma. It is noteworthy that in these experiments the platelets also remain very well preserved. By simple washing of the stromata with distilled water a distinct effect on them is produced. If a suspension of these were injected into rabbits and the animals killed after a definite time, thrombi composed of stromata were not only found to have arisen by the agglutination of these cell remnants, but also undoubted thrombi from blood-platelets were found. The increased agglutinability of the platelets must have been brought about by living substances produced by the solution of stromata in the blood plasma. If a suspension of stromata, which had been heated at 56 to $58 \mathrm{C}$. for forty minutes were injected, no such blood-platelet thrombosis ensues. So it seems that the living substances set free by the dissolving of the stromata in the plasma, are of great 
importance in effecting an increased agglutinability of the platelets. Experiments in animals which were allowed to live a longer period show that the stromata do dissolve in the circulating blood. In such experiments thrombi composed of stromata were found to have completely disappeared, so they must either have dissolved, or as is much less likely, have been washed away by the blood-stream. Thrombi composed of blood-platelets are also not seen in these animals, and from the fact that the individual plaques were still well preserved, it seems reasonable to suppose that they again enter the circulation.

Now let us consider how a foreign or heterologous serum acts. In Loeb's work, dog's serum was taken as the representative of strongly hemolytic and coagulating serums, while ox serum represented the group of strongly agglutinative serums. After the injection of dog's serum the rabbits died within a few minutes, showing characteristic symptoms (excitements, coma, convulsions, projecting eyeballs, abdominal distention, etc.). At the autopsy, fresh clotting was found in the lung arteries, in the right side of the heart, in the vena cava and in the lung veins, and death appeared certainly the result of these pathological findings. When, however, the experiment was carried out, after carefully opening the pericardium, it was seen that the clinical symptoms and the stoppage of breathing occurred while the heart continued to beat and the blood remained quite fluid. Microscopically, just as in the case of injection of a homologous serum, thrombi composed of stromata and platelets are to be seen in the lung capillaries, in the post-capillary venules, and also in the precapillary arterioles. But these thrombi differ from those discussed before in that the platelets show definite disintegration. Thus, not only agglutination, but destructive processes have been produced. This latter occurrence can only be caused by the heterologous serum itself, and the marked increase in the coagulability of the blood must also be referred to the disintegration of the platelets. One might be inclined to think that death might be produced by the blood-platelet thrombi, whereas the coagulation of the blood alone could not be the cause. But in this connection it is easy to show that where an animal has been given a nonlethal dose of dog's serum and been killed many hours later, capillary thromboses are just as marked as when the animal is killed at a muchi earlier stage. So, then, the fatal result can be referred neither to the blood coagulation nor to the capillary thrombosis. It must depend on some other changes in the blood, or increased viscosity of the plasma, or direct toxic action on the central nervous system.

The fact proved by Loeb's experiments with leech extract (hirudin) that the clotting is not responsible for death, was also confirmed by Kusama. Many rabbits, after injection of hirudin, died, although the blood postmortem was found quite fluid. The blood platelets seem to be 
diminished, since, according to all accounts, hirudin has a hindering action on the hemolytic and agglutinative properties of a heterologous serum. Experiments with ox serum give quite similar results. Kusama was unable to confirm the difference on which Loeb laid emphasis, namely, that by injection of ox serum marked clotting is absent. But he fully confirmed another point on which Loeb laid stress, the fact that agglutination plays a special rôle in all these experiments. In contrast to what occurs with dog's serum, many thrombi formed from stromata are found, but few composed of blood platelets. Also the platelets are not altered in the same way as after injection of dog's serum. It should be explained in addition that in some of the experiments with ox serum the coagulability of the blood did not show nearly so marked an increase as after injection of dog's serum.

Since as a matter of fact blood-platelet thrombosis, which in most researches has been given only a subsidiary place, is a result of hemolysis, we should be able to reproduce a similar thrombosis by injecting other hemolytic agents.

With this end in view, researches with ether were undertaken. It was previously believed that injection of ether was followed by a direct clotting of the blood, but as the result of experiments carried out by myself I have been opposed to this view for a long time. Then Loeb showed that injection of ether brought about an agglutination and destruction of erythrocytes, and considered the agglutination the cause of the death of the red cells. Our own experiments confirm this in so far that ether is hemolytic just like dog's serum, and brings about thrombosis by deposition of stromata and platelets. But no peculiar or special redcell thrombosis is called out by its injection. Ether is in addition directly destructive to the blood-platelets, and $s c$ the increased coagulability of the blood is explained. Glycerin and distilled water act in a manner similar to ether.

On the contrary, other blood-poisons, ricin for example, act in a surprising and entirely different way. Kusama's experiments showed that practically no hemolysis of erythrocytes occurred. Hence no noticeable thrombosis from blood-platelets or stromata ensued.

What does occur is really this : Ricin acts destructively on the myeloid and lymphoid cells of the spleen, bone-marrow and liver, and on the endothelial lining of the vessels. The fragments of cells so produced pack together in the capillaries of the spleen, bone-marrow and liver, and induce thrombosis. A definite increase in the coagulability of the blood does not occur as a rule. The coagulation of fibrin is secondary, just as in the case of thrombosis from stromata and blood-platelets.

Finally, experiments with bacterial cultures seem to be esp cually important. By injecting dead cultures of the Bacillus typhosus a definite 
fibrin throrubosis was produced, especially in the lung capillaries, but also in the capillaries of many other organs. In these thrombi leukocytes were enclosed, either broken down or well preserved. Destruction of myeloid and lymphatic cells was seen in the hemopoietic system, but not nearly to such a degree as after ricin. It was very strikingly brought out that these small thrombi from cell fragments (Trümmerthrombi) were accompanied by such a considerable deposit of fibrin. It seemed as if to explain it the bacteria must also be considered as foreign bodies. It is very difficult to extract the endotoxin of the Bacillus typhosus, so the experiment could only be repeated with typhoid bacilli heated for a much longer time. The results were similar, but not nearly so marked as when bacilli only killed by heat were injected. Also a previous immunizing of the animal against Bacillus typhosus had no obvious effect in decreasing the amount of thrombus building and of fibrin deposition. Dead cultures of the bacillus of dysentery and of staphylococci behave in exactly analogous fashion. In the case of cultures of the latter organism the destructive action on the cells of hemopoietic organs is very slight, whereas the formation of thrombi from leukocytes and fibrin in the capillaries of the lungs and other organs is very marked. The leukocytes always contained very numerous cocci.

In conclusion, I would say that the effect of bacterial injection is merely the production of leukocytic thrombi, and deposition of fibrin follows closely on this process. The definite occurrence of leukocytic thrombi, although they are only temporar", explains the marked leuko. penia which can be recognized one to four hours after the injection, in the peripheral blood, in most of the experiments.

To decide what part of the process could be referred to the mere presence of foreign elements in the blood, other animals were injected with a solution of India ink. The result was a definite thrombosis from blood-platelets in the lung capillaries. Many leukocytes were enclosed in the thrombus, but no deposit of fibrin occurred. This result is different from that produced by bacteria, in that deposition of fibrin was absent, a blood-platelet thrombosis being the chief result. No marked destruction of leukocytes was seen. Phagocytosis of particles of India ink, although it was present, was not so marked as in the case of cocci. The packing of the leukocytes inside the lung capillaries produced a leukopenia just as after injection of dead bacterial cultures.

Collargol was also injected and produced a thrombosis chiefly from blood-platelets, agglutination of leukocytes being practically absent. Olive oil gave a similar picture. Injection of ground rice was followed by the same result induced by bacteria, in that, whereas blood-platelet thrombosis did not occur, thrombi from leukocytes and fibrin were to be found in the lung capillaries. 
Thus all foreign elements do not act in the same way. Substances like India ink, collargol and olive oil must have some attraction for plateiets, whereas the ground rice and dead bacteria have none. The first three produce thrombosis from blood-platelets, the latter groups only leukocytic thrombosis and the greater the destruction of leukocytes and platelets the sooner is fibrin deposited.

\section{CONCLUSIONS}

What result, then, can we glean from all these experiments? We have seen that different poisons, whether hemolytic in action or not, bring about distinctive forms of capillary thrombosis, among which we can differentiate the following types:

I. Blood-Platelet Thrombosis.-This arises by intravenous injection of a homologous serum, of solutions of hemoglobin and stromata from: homologous blood, of heterologous serums, ether, glycerin, distilled water, India ink, collargol and olive oil. The thrombi arise in the capillaries of lungs, liver and spleen.

II. Thrombosis from Blood-Stromata.-This occurs in pure form only, when very marked hemolysis occurs in the blood-stream as a result of the action of hemolytic agents. Otherwise it occurs only in conjunction with blood-platelet thrombosis.

III. Thrombosis from Fragments of the Blood Elements (Bluttrümmerthrombose).-Disintegration of the elements of the blood occurs by introduction of ricin, bacterial toxins, etc., which destroy the leukocytes, lymphocytes, macrophages of the spleen, liver and bone-marrow.

The fragments, in conjunction with scanty fragments of erythrocytes, roll themselves together to form thrombi in the spleen and liver. Fibrin is then subsequently deposited there.

IV. Leukocytic Thrombosis.-The polymorphic and mononuclear leukocytes phagocyte the foreign elements introduced into the bloodstream, and are stopped and held back in the capillaries of the internal organs, especially the lungs, spleen and liver, so that a leukopenia results in the peripheral circulation. This process is especially brought out by bacteria, but also by various indifferent foreign substances like particles of ink and starch.

V. Fibrin Thrombosis.-Although by injerting stromata or foreign serums the coagulability of the blood can be increased, no deposit of fibrin occurs. Deposit of fibrin occurs only secondarily, as a sequel of the types of thrombosis already described.

Let us take, then, a final glance over the subject of static and toxic thrombosis. We see that neither endothelial damage, on which so much stress was previously laid, nor a direct coagulation of the blood, play any rôle. Only two real reasons for the thrombosis require to be con- 
sidered, and their merits weighed one against the other. I refer to the changes in the blood elements themselves, and to the slowing of the bloodstream. Neither of these two factors can be excluded, as even in the experiments where thrombi of different sorts are produced by heightening the agglutinability of the blood-platelets by the disintegration of white or red corpuscles, these thrombi can occur only where normally a marked slowing of the blood-stream is present; for example, in the capillaries of the lungs, liver and spleen. But even by very marked destruction of the blood elements it is impossible to produce thrombi by deposition in the larger veins when no slowing of the current occurs.

An additional factor, therefore, needs to be considered, especially in cases in which the thrombi are localized in the large veins, and where the above-mentioned capillary regions of the lungs, liver and spleen generally implicated in toxic thrombosis remain free. In such a case the increased agglutinability of the blood-platelets cannot be the direct exciting cause, but a definite and recognizable slowing of the blood-stream must first of all occur.

So we see from all these experiments that the slowing of the bloodstream and the alteration of the blood elements themselves, especially alterations of the platelets, are the chief factors in the production, not only of the static, but of toxic thrombosis, so far as we are concerned with thrombus formation in the flowing blood-stream. In static and similar types of thrombi the slowing of the blood-stream is of prime importance, while for the toxic varieties the changes in the blood elements have the dominating influence.

Nevertheless, we must ask ourselves whether very important and intimate relationship do not exist between thrombosis and coagulation of fibrin. 'There is no doubt that, with a few exceptions, coagulation of fibrin as a rule sooner or later inevitably follows thromboses. So the division of the process of thrombosis, as set forth by Loeb for the lower animals, is now quite clear. 'The first stage is the erection of a morphological structure by a process of agglutination. Fibrin ferment is obtained from the agglutinated elements and cements them together by coagulation. This is the second phase.

Therefore, in the process of thrombosis in human beings the phylogenetic development of the complicated mechanism of coagulation is repeated. 